reinfection at sites where pathogens will often first enter the body ${ }^{6}$. Whether these different types of memory $\mathrm{T}$ cell arise through the same pathway remains to be determined.

These two studies suggest that a goal of vaccine design should be to stimulate a large, robust response from the effector $\mathrm{T}$ cells from which memory T-cell populations can arise. However, the conditions that best promote effector cells to become memory cells still need to be fully defined. Therapeutic targeting of the DNA-modification machinery, such as Dnmt3a, might prove to be a useful strategy to increase vaccine efficacy.

Kyla D. Omilusik and Ananda W. Goldrath are in the Division of Biological Sciences, University of California, San Diego, La Jolla, California 92093, USA.

e-mail:agoldrath@ucsd.edu
1. Akondy, R. S. et al. Nature 552, 362-367 (2017).

2. Youngblood, B. et al. Nature 552, 404-409 (2017).

3. Kaech, S. M. \& Cui, W. Nature Rev. Immunol. 12 , 749-761 (2012).

4. Winter, D. R., Jung, S. \& Amit, I. Nature Rev. Immunol. 15, 585-594 (2015)

5. Gray, S. M., Kaech, S. M. \& Staron, M. M. Immunol. Rev. 261, 157-168 (2014)

6. Chang, J. T., Wherry, E. J. \& Goldrath, A. W. Nature Immunol. 15, 1104-1115 (2014).

This article was published online on 13 December 2017.

\title{
Martian water stored underground
}

\section{Why did Mars lose so much of its surface water, whereas Earth retained its? Models of the evolution of minerals on the two planets suggest one explanation: the Martian water was drawn into the planetary interior. SEE LETTER P.391}

TOMOHIRO USUI

$\longrightarrow$ bservations of Mars and its geology suggest that the red planet once had an Earth-like hydrological cycle that included large lakes or oceans. In contrast to this ancient wet environment, the surface of Mars today is cold and dry. The transition to this present state is closely linked to the fate of the planet's surface water, which is poorly understood. A substantial amount of surface water escaped to space from the atmosphere, in part because of Mars' relatively low gravity ${ }^{1}$. However, atmospheric-escape models account for only some of ancient Mars' surface water. On page 391, Wade et al. ${ }^{2}$ propose that much of the surface water was sequestered underground.

Erupted lava had the chance to interact with surface water to form hydrated crust on both Earth and ancient watery Mars. Wade et al. examine the thermodynamic properties of the water-bearing 'mafic' crusts (which largely consist of the rock basalt) of each planet, and show that Martian basalt can hold more water than terrestrial basalt, and can effectively transport it to a greater depth below the surface (more than 90 kilometres; Fig. 1). The authors also compute the stability of water-containing minerals in hydrated crusts and their bulk-rock densities along both planets' geotherms (which describe how temperature varies with depth). They conclude that the burial of hydrated crusts progressively hydrates the interior of Mars, but that this process does not work effectively for Earth.
The four rocky planets of the Solar System (Mercury, Venus, Earth and Mars) are thought to have been formed by the accretion of similar planetary building blocks. This resulted in their mantles having broadly similar compositions of all the major elements, except for iron. Metallic iron partitions into the metallic cores of the rocky planets, whereas iron(II) oxide accumulates in silicate-rich planetary mantles. A thermodynamic property known as oxygen fugacity, which is a measure of the amount of oxygen present in a mixture, controls iron content in planetary basalts. Martian basalts have

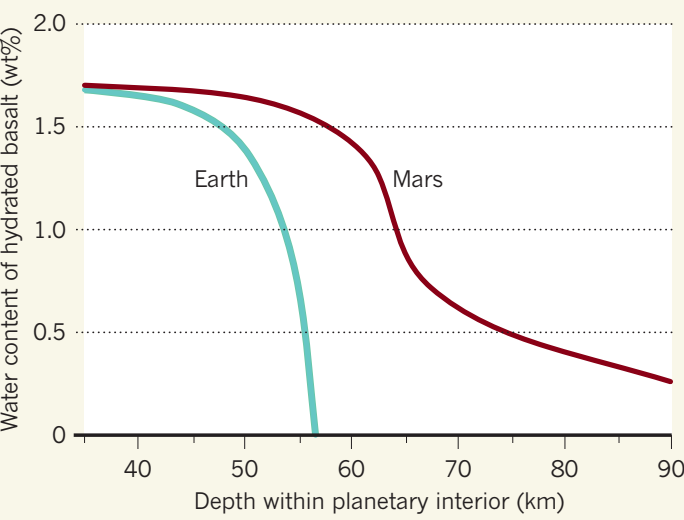

Figure 1 | The water content of basalt on Earth and Mars. Wade et al. ${ }^{2}$ report modelling of the surface evolution of Earth and Mars, which allowed them to estimate the amount of water that can be sequestered by basalt rock in the planets' interiors. They find that the Martian basalts can store more water (shown as percentage by weight), and at greater depths, than their terrestrial counterparts. This suggests that much of the water thought to have existed on the surface of ancient Mars was sequestered underground. (Adapted from Fig. 1a of ref. 2.) a distinctly higher content of iron(II) oxide (about $17 \%$ by weight) than do typical basalts on Earth (about $7-10 \%$ by weight), which suggests that oxygen fugacity was higher during Martian core formation than it was during Earth's core formation. Wade and colleagues show that this compositional difference, along with the different geotherms of Mars and Earth, has a key role in the storage and transportation of planetary surface water in the crust and mantle of the two planets.

Hydration processes generally expand the crustal volume, making the crust less dense. By contrast, Wade and colleagues' thermodynamic modelling indicates that the iron-rich Martian basalts undergo small volume expansions during hydration. Furthermore, hydrated iron-rich Martian basalts tend to melt at lower temperatures (about $800-900^{\circ} \mathrm{C}$ ) than does anhydrous mafic rock (about $1,200^{\circ} \mathrm{C}$ ), and this melting leaves relatively dense hydrated residues in the mantle. In the apparent absence on Mars of tectonic processes that recycle crust material into the mantle, the authors propose that successive burial of hydrated crust might have induced hydration of the mantle - such burial would have gradually increased the temperature and pressure applied to hydrated crustal basalts, causing them to melt, and therefore to leave hydrated residues in the mantle.

Hydration of the Martian mantle would also lead to it becoming more oxidized. However, geochemical analysis of meteorites (known as shergottites) formed from young Martian basalts suggest that their source in the mantle is less oxidized than Earth's mantle ${ }^{3}$. Moreover, the shergottite source is depleted in water (less than 50 parts per million $)^{4}$ relative to Earth's mantle (typically about $100-200$ p.p.m.). How do these observations fit into Wade and colleagues' suggestion that the Martian mantle contains more water than Earth's? Experiments have shown ${ }^{6}$ that the shergottite source is located at a depth of approximately $100 \mathrm{~km}$, which is near the base of the hydrated mantle column proposed by Wade and co-workers. The lower oxidation and hydration of the shergottite source might therefore be representative of (or place 
a cap on the state of) the uppermost region of the unhydrated mantle, unless it represents a local phenomenon.

The global surface-water inventory of Mars was originally estimated to be about $2 \times 10^{7}$ to $2 \times 10^{8} \mathrm{~km}^{3}$ (ref. 7 , and references therein), on the basis of the size of the putative ancient oceans. But estimates of the total water loss to space are much smaller (less than $10^{7} \mathrm{~km}^{3}$, based on atmospheric-escape models ${ }^{7}$ ). The discrepancy between these estimates hints at the existence of a 'missing' water reservoir beneath the surface. The widespread distribution of hydrated materials on the surface of Mars also implies the existence of a crustal water reservoir, but conventional spectroscopic observations are able to see only the surface veneer. Wade and colleagues' thermodynamic modelling, together with remotesensing observations, offers a means to work out the depth profile of hydrated materials, and to calculate a reasonable estimate of the volume of the crustal water reservoir.

Ground ice might also account for the missing water reservoir on Mars ${ }^{8-10}$. Subsurface radar-sounder measurements ${ }^{8}$ have detected an anomaly in an electrical property of rocks in the planet's northern hemisphere, which implies that massive ice deposits are embedded among or between layers of sediment and volcanic materials at a depth of $60-80 \mathrm{~m}$. The ground-ice model has also been proposed on the basis of analyses of hydrogen isotopes in Martian meteorites ${ }^{9}$ and of crater morphology ${ }^{10}$. The crater study indicates that the subsurface water ice has a volume of about $3 \times 10^{7} \mathrm{~km}^{3}$, which is comparable to the size of the ancient oceans. Subsurface exploration will be required to test both the hydratedcrust and ground-ice theories, and therefore to shed light on the evolution of the Martian water inventory.

Tomohiro Usui is at the Earth-Life Science
Institute, Tokyo Institute of Technology,

Tokyo 152-8551, Japan.

e-mail:tomohirousui@elsi.jp

1. Lammer, H. et al. Space Sci. Rev. 174, 113-154 (2013).

2. Wade, J., Dyck, B., Palin, R. M., Moore, J. D. P. \& Smye, A. J. Nature 552, 391-394 (2017).

3. Herd, C. D. K. et al. Geochim. Cosmochim. Acta 66, 2025-2036 (2002).

4. Usui, T., Alexander, C. M. O'D., Wang, J., Simon, J. I. \& Jones, J. H. Earth Planet. Sci. Lett. 357-358, 119-129 (2012).

5. Saal, A. E., Hauri, E. H., Langmuir, C. H. \& Perfit, M. R. Nature 419, 451-455 (2002).

6. Filiberto, J. \& Dasgupta, R. J. Geophys. Res. Planets 120, 109-122 (2015).

7. Kurokawa, H. et al. Earth Planet. Sci. Lett. 394, 179-185 (2014).

8. Mouginot, J., Pommerol, A., Beck, P., Kofman, W. \& Clifford, S. M. Geophys. Res. Lett. 39, L02202 (2012).

9. Usui, T., Alexander, C. M. O’D., Wang, J., Simon, J. I. \& Jones, J. H. Earth Planet. Sci. Lett. 410, 140-151 (2015).

10.Weiss, D. K. \& Head, J. W. Icarus 288, 120-147 (2017).

\section{Tumour lymph vessels boost immunotherapy}

A high level of expression of the growth-factor protein VEGF-C is associated with tumours that have extensive lymph vessels and poor prognosis. It emerges that such tumours are highly susceptible to immunotherapy.

\section{CHRISTINE MOUSSION \\ \& SHANNON J. TURLEY}

A dvances in our understanding of tumour immunology have led to immense clinical interest in harnessing immune cells to target cancer ${ }^{1}$. The immune system's killer (cytotoxic) T cells can search for and destroy abnormal cells that they encounter while patrolling tissues. However, in the suppressive microenvironment of a tumour, such cells often enter a dysfunctional state. A goal of cancer immunotherapy is to trigger or revitalize the antitumour immune response either through vaccination strategies or by using an approach called checkpoint-blockade therapy that dampens immuno-inhibitory signals, such as those of the PD-1 or CTLA-4 pathways ${ }^{2}$. Writing in Science Translational Medicine, Fankhauser et al. ${ }^{3}$ show that an increase in the expression of a protein that promotes tumour growth can also make a tumour more responsive to immunotherapy.

Tumours need a vascular supply to grow. Blood vessels bring nutrients and oxygen to a tumour, whereas lymphatic vessels remove fluid and waste. A tumour and the cells in its surrounding milieu stimulate the development

of these two branches of the vascular system by secreting proteins that are members of the vascular endothelial growth factor (VEGF) family ${ }^{4}$.

The protein VEGF-A promotes the generation of new blood vessels (angiogenesis). Decades of work aimed at impairing tumour growth by blocking angiogenesis has led to the approval of drugs that inhibit signalling by members of the VEGF family, with most approaches $^{4}$ target-

"Anincrease in the expression of a protein that promotes tumour growth can also make a tumour more responsive to immunotherapy.' ing VEGF-A.

VEGF-C promotes lymphatic-vessel formation (lymphangiogenesis) by signalling through the VEGFR3 receptor ${ }^{5}$. Lymphatic vessels can promote the process of metastasis - tumourcell spread beyond the primary tumour growth site - by providing a route for cancer cells to exit a tumour and reach nearby structures termed lymph nodes. This process enables tumour cells to grow in lymph nodes, and to disseminate from there to other locations. Many tumours associated with metastasis, such as melanoma and breast cancer, express high levels of VEGF-C and contain a dense network of lymphatic vessels ${ }^{6}$. Moreover, an increase in VEGF-C expression in tumours is highly correlated with metastasis to lymph nodes, and with a reduction in survival in individuals with different tumour types, including skin, breast and lung cancers ${ }^{7}$.

Does an increase in tumour lymphangiogenesis always promote tumour growth? Fankhauser and colleagues addressed this question by analysing the role of VEGF-Cdriven lymphangiogenesis in tumour growth. They studied mice that express high levels of VEGF-C and provide a model system for studying cancer. When the researchers treated these animals with VEGF-C-blocking antibodies, they observed a surprising result: although high VEGF-C expression is associated with poor tumour prognosis, VEGF-C inhibition in the context of tumour immunotherapy resulted in increased tumour growth. This suggested that VEGF-C might have another, previously unsuspected role in limiting tumour growth by boosting patient responses to immunotherapy.

The authors investigated the basis of this phenomenon. They found a strong positive correlation between VEGF-C levels and the strength of a tumour-specific cytotoxic T-cell response after immunotherapy in their model mice. They also observed a similar association between high VEGF-C expression and boosted immunotherapy responses (Fig. 1) when analysing data from people who have a metastatic form of skin cancer called melanoma. Together, these results suggest that VEGF-C and the lymphatic system might have unexpected roles in aiding cancer immunotherapy. Perhaps VEGF-C levels could be used as a biomarker to identify patients who are likely to respond to immunotherapy. 